\title{
ORGANIC DYSLALIA
}

By ALICE RUMMEL, B.A. Log Rand

Speech is the highest and most fundamental human attribute and without it one is set outside the possibilities of constant communication with his fellows. Speech is also an important tool for social adjustment, and must conform to the environment.

When listeners recognise speech as defective, impaired social relationships arise which further inhibit and distort speech. As the defect gets progressively worse the individual's social relationships become impaired. Thus it is not sufficient to know only what kind of defect the person has, but also what kind of person has a speech defect. The spcech defect alone has no meaning but must be considered in relationship to the whole personality, since speech and personality are closely linked.

There are four aspects necessary for the production of normal speech:

1. Phonatory - concerned with the voice or laryngeally controlled air siream.

2. Articulatory - here the stream is modified by articulatory organs of the mouth and throat.

3. Linguistic - which must conform to an arbitrary code of meanings for communication.

4. Auditory.

In these processes of vocal control and articulatory modification, the chief guide "as to the accuracy of conformity to the code is the oral communication of others, this interpretation being an integral part of the function of speech."

Many faculties are concerned in the completion of this fourfold picture. If one fails to develop, speech fails, even though the other functions are normal. Psychologically, articulation, which is the moulding of speech sounds, is not a special faculty in itself, but is rather an amalgamation of several abilities that develop side by side during the first years of life. It is largely these abilities that determine the onset of speech. If one of these abilities are late in developing, speech will be delayed, defective or both. The factors underlying articulation are both sensory and motor. They are:-

1. Discrimination and acuity to sounds of high frequency.

2. Memory span for individual speech sounds.

3. Speed of muscle movement.

4. Specialization of movement.

Froeschels and Jellinek define Dyslalia as con. cerning "all disorders occurring in articulation. If sounds are produced incorrectly or replaced by others, or if they are entirely lacking, then the person is dyslalic". The classification of Dyslalia is mainly based on etiology. There are three main divisions:

A. Organic.

B. Functional.

C. Psychological.

These divisions, however, niay overlap and are interrelated, and have no clcarly defined boundaries. There is seldom an organic factor alone, but usually there is some introduction of a psychological factor.

Organic Dyslalia presupposes structural deformity or abnormality and thus implies a disorder of articulation due directly to the fact that some part or parts of the articulatory apparatus are so defective anatomically and physiologically that they are unable to perform their primary functions of chewing, sucking and swallowing, or their secondary derived function of articulating with a great degree of perfection.

There are any number of possible oral deformities, e.g. cleft lip, paralysis of the lip or lip inadequacy due to shortness, trauma or other cause; abnormalities in size, symmetry or func. tional efficiency of the tongue, tongue-tie, growths on the tongue and paralysis; disturbed dental relationships such as all types of malocclusion, spaced or missing, misplaced or supernumerary teeth or other dental conditions that may interfere with usual articulatory adjustments necessary for good speech production; cleft palate, abnormally highly arched palate, and short cleft or paralysed velum. These are some possible causes of organic dyslalia .

A deformity of any one of the structures of the oral region tends to cause a distortion of the other adjacent structures, since their anatomical and functional relationships are so close. Because of these close relationships, the condition and functioning of the oral structures should be carefully noted, in order to determine their possible role in causing the given speech defect. If the basis of the speech defect is organic, surgical and medical attention may be needed; if it is functional or psychological in origin, the treatment should proceed from the approach indicated.

\section{EXAMINATION PROCEDURES}

Van Riper states that the mouth and throat of all cases should be examined. Although the case may effect a good acoustic result of speech in spite of a physical anomaly, such an organic abnormality will be some handicap in speech 
development. The presence of an organic abnormality is not important unless it stands in functional relationship to defective speech sounds. Diagnosis should not be made hastily. Other causal factors may also be of great importance besides the organic defect.

Each part of the articulatory organs should be examined thoroughly. The structure itself should be examined not only in quiescence but also in its relation to the appropriate speech sounds. All evidence of a handicapping abnormality and all compensatory movements should be recorded. Other factors such as intelligence, auditory acuity, motor inco-ordinations and especially emotional history and background of the case should be taken into consideration.

Defects caused by mouth deformities are usually structural in origin. However, the deformity of the mouth is not always the explanation for the speech defect. Thus:

1. Structural anomalies of organs of articulation may occur but there is no speech defect.

2. Acceptable speech can be produced by adjustments of the articulatory organs which are mechanically quite different from adjustments usually made in speech.

3 . There is more than one way of producing a particular speech sound. For example, in the production of the " $\mathrm{t}$ " sound, if the patient cannot clevate the tip of the tongue he can use the blade.

The severity of the speech involvement depends upon the extent of the abnormality, and also upon the degree of compensation by the articulatory organs before the restoration of the function of the imperfect structures has been achieved. The ability to compensate differs with every individual case even if the extent of the deformity is similar. This difference may depend on differences in auditory awareness of speech peculiarity, kinesthetic sensitivity, intelligence, motivation and environmental stimulation.

Van Riper suggests that bright children from homes with high speech standards will learn compensatory methods more easily than those less inligent children from homes with poor speech standards. This ability to compensate involves the child's residual diathesis or X-factor.

The commonly-accepted postulate is that the usual adjustment of the articulators used for a given sound is the easiest, but by no means the only possible adjustment by which the given sound may be produced. Although a person has a structural abnormality and cannot employ the usual manner of articulation, he may learn a new method for producing the sound, thus developing a unique skill. Thus his structural abnormality does not interfere with his speech. But if the individual lacks this ability to acquire unique skills, his speech will be defective. In his speech difficulty then due to his structural anomaly or to his lack of facility?

This facility is complex — "made up of mutually supporting factors such as the individual's health, endocrine drive, general vitality, intelligence, hearing acuity, efficiency of motor co-ordination, motivation and training for good speech afforded by his social environment." If these factors are in his favour, he can make successful compensations for any marked structural deviations. If these factors are against him a slight deviation may prove more of an obstacle than he can overcome without special help.

This is not the ONLY important factor in the individual with congenital malformations and other structural abnormalities. These anomalies themselves may be a specially, narrowly localized deviation and present an uncomplicated problem, or the anomaly may be evidence of a constitutional inferiority, with characteristic reduction of impetus in any project which requires individual initiative and stamina. Thus in drawing up a programme for speech rehabilitation, this $\mathrm{X}$ factor must be taken into consideration. It should be considered in the broad spheres when evaluating the child, not only in his ability to learn speech, but also his reactions in meeting other demands for initiative, resourcefulness and stamina.

Van Riper says that there are two methods for minimizing the influence of organic abnormalities: 1. Reconstruction of defective organic structure through surgery and orthodontia.

2. Teaching of compensatory movements in speech sound production.

Orthodontia has seen great advancement and changes in dental, palatal and jaw structures have now been accomplished. Cases, especially children with marked mouth deformities should be referred to the appropriate specialists so that reconstruction work can be carried out. Thus effort will not be wasted in teaching compensatory movements where medical or dental care should first be instituted. Palatal abnormalities are frequently associated with those of the jaws and orthodontic projection or retraction of the jaw can facilitate tongue contact with the roof of the mouth.

A wide variety of surgical repair and construction work can be carried out, e.g. scar tissue can be excised and grafts can be made which will provide the necessary mobility. High palatal arches can be lowered and the velum can be modified. The most desirable time for surgery is during childhood. The speech therapist should recommend to the parents that it be done and see that it is carried out. The paralyzed structures can occasionally be helped by exercise adminis- 
tered by a physiotherapist. There is usually recourse to more biological functions and tying up of the specialized movement with gross muscular action. The case may also need a hearing aid.

The teaching of compensatory movements can be initiated if the case cannot be helped by surgery or orthodontia. Since all speech sounds may be produced in a variety of ways, e.g. the art of the ventriloquist demonstrates compensatory activity of that of the lips and jaws. Normal speakers may have profound anatomical abnormalities and produce sounds with a perfect acoustic end result.

One must take into account and try to minimize the following factors in teaching compensatory movements:

(i) complexity of performance (the fewer adjustments the better) ;

(ii) ease of transition from other sounds;

(iii) amount of facial distortion;

(iv) distinctness of kinesthetic and tactual sen. sations;

(v) motivation and co-operation of the subject;

An organic anomaly often causes unfavourable emotional and social attitudes. The reason for this is that any peculiarity of appearance, behaviour or speech tends to interfere with favourable adjustment. These attitudes may be prevented or minimized by adequate structural repair before the child can become sensitive about his appearance. Reactions to deformities should be made objective. Let the child face his problem squarely and learn to deal with any difficulties which he may encounter. On the other hand, the child's abnorm. ality must not be kept in consciousness all the time. Parents and teachers should stress other social graces, e.g. good grooming, friendly manneriams, conversational ability, music, etc.

\section{THERAPY :}

All pessible medical, surgical and orthopraxic procedures must be tried as early in childhood as possible because:

1. It prevents fixation of defective motor patterns in the nervous system, which will be displaced only with great difficulty, if at all.

2. It allows deformed parts an opportunity of reshaping into a normal mould after surgical repair by means of the natural processes of growth.

3. It brings cosmetic improvement very early and therefore avoids the social insecurity and selfconsciousness brought about by physical deformities.

If normal structure has not been obtained through medical, surgical or dental care, training should begin as early as possible to teach the child functional compensations for his structural deficiencies, and differences from the normal.

"Mechanically, standard speech is dependent upon normal anatomical structure," When anatomy is not normal, functional compensations should imitate as nearly as possible the mechanics of the correct sound rather than its placement in the mouth.

After successful surgical repair, previous compensations must be eliminated, unless they are mechanically efficient in the production of the desired sounds. An isolated sound may be formed in a non-standard way with some facility, but when combined with other sounds or into blends these non-standard methods may not produce the desired acoustic result. Thus the compensatory method must be considered as to wether it can be used together with other sounds, i.e. its usefulness for ordinary conversational speech.

If structural anomalies of teeth, tongue and mouth remain, after surgery, compensations must be developed. The decision should only be made after careful analysis based on knowledge of mechanical properties. However, one must keep in mind the individuality of each case.

Thus in a programme of treatment and training the surgical repair comes first. The case can then proceed to exercises to strengthen the parts required, and go on to the correction of defective sounds.

The prognosis depends on the severity of the deformity and on the efficiency of repair and amount of speech training. The operation alone may have no noticeable effect on his speech. Postoperative training is a great deal more than half of his speech rehabilitation. Complete or even partial surgical repair may be impossible. Also it may be impossible to wholly compensate for any deficiencies by mechanical means. This needs special speech training. Often the very badly deformed cases require not so much direct speech training as mental hygiene.

\section{CONCLUSION.}

In this article an attempt has been made to cover some aspects in the field of organic dyslalia. Details of the many defects of organic dyslalia have been avoided. Instead a short resume indicating some important aspects of the field have been given.

The importance of organic factors is obvious, and should not be overlooked. nor must too much emphasis be laid on them, because an individual may display normal speech in spite of organic abnormality. 